\title{
Coeficientes de Herdabilidade e Correlações Genéticas para as Produções de Leite e de Gordura, em Diferentes Níveis de Produção, para Raça Holandesa no Estado do Rio Grande do Sul
}

\author{
Tomás Weber ${ }^{1}$, Paulo Roberto Nogara Rorato², Gilka Berenice Barbosa Ferreira², Arione \\ Augusti Boligon ${ }^{3}$, Diego Ghedini Gheller ${ }^{3}$, Luiz Felipe Waihrich Guterres ${ }^{4}$
}

RESUMO - Para estudo da heterogeneidade de variâncias e estimação de coeficientes de herdabilidade e de correlação genética em diferentes níveis de produção de leite e de gordura, foi utilizado um conjunto de dados composto de 8.887 registros de produção de 5.875 vacas filhas de 1.125 touros, no período de 1984 a 2001. Os dados foram estratificados em três diferentes níveis de produção, de acordo com a média de produção de leite do rebanho, em: baixo ( $\leq 6000 \mathrm{~kg}$ ), médio ( $>6000 \mathrm{a} \leq 7500 \mathrm{~kg})$ e alto (>7500 kg). Os componentes de (co)variância foram obtidos pelo Método da Máxima Verossimilhança Restrita, utilizando um modelo animal, incluindo, como efeitos aleatórios, os efeitos genéticos direto do animal e o de ambiente permanente e, como fixos, rebanho dentro do núcleo de controle leiteiro, ano/época do parto e ordem da lactação, além das covariáveis idade da vaca ao parto e duração da lactação. As médias observadas e respectivos desvios- padrão, para produção de leite (PL) e produção de gordura (PG) variaram de $4.922 \pm 743 \mathrm{~kg}$ a $8.760 \pm 126 \mathrm{~kg}$ e de $163 \pm 30 \mathrm{~kg}$ a $298 \pm 53 \mathrm{~kg}$, respectivamente. As estimativas de herdabilidade variaram de 0,13 a 0,19 e de 0,16 a 0,21 , respectivamente para PL e PG. Os níveis que apresentaram menores estimativas de herdabilidade para produção de leite e gordura foram o baixo e o médio, sugerindo que, nestes níveis, a resposta esperada pela seleção poderá ser menos eficiente. Os coeficientes de correlação genética entre PL e PG variaram de 0,91 a 1,00, indicando que a seleção realizada para uma das características promoverá resposta quase que equivalente na outra.

Palavras-chave: heterogeneidade de variâncias, parâmetros genéticos, produção de gordura, produção de leite

\section{Heritability Coefficients and Genetic Correlations for Milk and Fat Yields at Different Production Levels for Holstein Breed in the State of Rio Grande do Sul}

\begin{abstract}
Heritability and genetic correlations coefficients in different levels of milk production and fat were evaluated in this study. A data set of 8,887 milk and fat records from 5,875 Holstein cows, sired by 1,125 bulls from 1984 to 2001 were used. Data were stratified in three levels according to the herd milk production, low $(\leq 6000 \mathrm{~kg})$, medium $(>6000 \mathrm{a} \leq 7500 \mathrm{~kg})$ and high $(>7500 \mathrm{~kg})$. The (co)variance components were estimated by Restricted Maximum Likelihood Method including in an animal model as random, the genetic direct and the permanent environmental effects and as fixed, the effects of herd: milking control nucleus, year season, lactation order, and the age of the cow at parturition and the period of lactation as co variables. The observed means and standard deviations for milk (PL) and fat (PG) yields ranged from $4,922 \pm 743 \mathrm{~kg}$ to $8,760 \pm 126 \mathrm{~kg}$ and from $163 \pm 30 \mathrm{~kg}$ to $298 \pm 53 \mathrm{~kg}$, respectively. The estimated heritability coefficients ranged from .13 to .19 and from .16 to .21, respectively for PL and PG. The highest values for the heritability coefficients, for PL and PG were observed at the low and medium levels, suggesting that at these levels the improvement expected by selection could not be reached. The genetic correlation coefficients ranged from .91 to 1.00, suggesting that the selection for one of the characteristics will produce a similar response in the other.
\end{abstract}

Key Words: genetic parameters, fat production, milk production, variances heterogeneity

\section{Introdução}

A fim de se obter melhores índices de produção na pecuária leiteira, é comum a introdução de animais da raça Holandesa nos rebanhos leiteiros brasileiros. Entretanto, apesar da participação expressiva desta raça, pura ou por meio de cruzamentos, no total de leite produzido em nosso país e, também, considerando que a média da raça em nosso meio vem aumentando ano após ano, ainda há muito que se estudar a respeito dos aspectos genéticos desta população em nossas condições de criação. O conhecimento de

\footnotetext{
1 Aluno do curso de Zootecnia, bolsista FAPERGS-Campus Universitário, CEP:97105-900, Santa Maria, RS. E.mail: tomasweber@bol.com.br

2 Prof. Dr. Departamento de Zootecnia da Universidade Federal de Santa Maria.

${ }^{3}$ Aluno de curso de Zootecnia, bolsista CNPq/PIBIC.

${ }^{4}$ Aluno de Pós-graduação em Zootecnia da Universidade Federal de Santa Maria.
} 
aspectos genéticos de uma população é essencial para a obtenção de informações que possam orientar os produtores e técnicos na identificação e no acasalamento de animais geneticamente superiores, visando maior progresso genético nos programas de seleção. Para a realização da seleção é preciso ter conhecimento dos valores dos componentes de (co) variância, para que sejam determinados os parâmetros genéticos para as características a serem selecionadas.

A seleção e o uso de animais geneticamente testados têm conduzido, ainda que de forma lenta, a mudanças positivas nas médias das características produtivas (Bastos et al. 1998). A obtenção de ganhos genéticos mais significativos está diretamente relacionada ao conhecimento do comportamento das variâncias em ambientes diferentes, uma vez que a magnitude do coeficiente de herdabilidade está relacionado ao da variância genética aditiva.

Segundo Torres (2000), quando a heterogeneidade das variâncias em ambientes diferentes é desprezada, se presente, a produção das filhas de determinado reprodutor será ponderada na proporção dos desviospadrão dos rebanhos em que elas foram criadas; o resultado é que as produções das filhas oriundas de rebanhos com maiores variâncias influenciarão mais a avaliação de reprodutores que as produções de filhas oriundas de rebanhos com menores variâncias. De acordo com Torres (2000), no caso das filhas dos diversos reprodutores serem distribuídas, uniformemente, entre os rebanhos com variação diferente, nenhum vício nas avaliações seria observado. Entretanto, se as herdabilidades também diferem entre rebanhos, a acurácia da avaliação genética seria reduzida, em razão da não-consideração destas diferenças. Segundo Ramos et al. (1996), uma conseqüência direta da heterogeneidade das variâncias em diferentes níveis de produção dos rebanhos é o risco de se selecionar maior proporção de animais de maior variabilidade fenotípica e não de maior valor genético, podendo, com isso, ocorrer até redução no progresso genético esperado.

Com o objetivo de estudar o efeito da heterogeneidade das variâncias sobre a avaliação genética e a classificação de reprodutores, pesquisadores como Hill et al. (1983), Boldman \& Freeman (1990), nos Estados Unidos da América (USA), Stanton et al. (1991) estudando rebanhos na Colômbia, no México e em Porto Rico e Araújo et al. (2002), no Brasil, encontraram estimativas de variâncias heterogêneas de acordo com o aumento no nível de produção dos rebanhos e com as diferentes regiões e países e relataram aumento na magnitude das estimativas, com o aumento do nível de produção dos rebanhos, sugerindo que os coeficientes de herdabilidade seriam maiores e os ganhos por meio de seleção mais significativos nos níveis mais altos de produção.

A estimação dos componentes de variância para as características de produção em gado de leite tem incluído pesquisas sobre homogeneidade de variância entre classes de rebanho-ano-estação, que vêm fornecendo considerável evidência da existência de variâncias heterogêneas entre os diferentes níveis de produção. Boldman \& Freeman (1990), estudando lactações de vacas da raça Holandesa, nos USA, observaram componentes de variância genética aditiva e residual crescentes com os níveis de produção. Lee \& Park (1994), avaliando dados de produção de leite de rebanhos estratificados em grupos de baixa, média e alta produção, na Coréia do Sul, encontraram variâncias heterogêneas nos três níveis de produção de leite estudados. Marion et al. (2001), trabalhando com animais da raça Holandesa, no Rio Grande do Sul (RS), verificaram falta de homogeneidade nos componentes de variância estimados. Com base no desvio padrão fenotípico da produção de leite, para a raça Pardo-Suíça no Brasil, Araújo et al. (2002) estratificaram os rebanhos em três classes e verificaram que os componentes de variância aumentaram da classe de baixo para a de alto desvio-padrão, caracterizando a heterogeneidade de variância entre os níveis.

A heterogeneidade dos componentes de variância se reflete diretamente nas estimativas dos componentes de herdabilidade. Hill et al. (1983), Boldman \& Freeman (1990), Mirande \& Van Vleck (1985), Lofgreen et al. (1985) e Dong \& Mao (1990), estudando registros de produção de vacas da raça Holandesa, encontraram tendência de avanço na magnitude dos coeficientes de herdabilidade com o aumento na média de produção, enquanto Ramos et al. (1996), no Brasil, trabalhando com animais da mesma raça, em diferentes níveis de produção (baixo, médio e alto), estimaram coeficientes de herdabilidade inferiores no nível médio $(0,24$ para produção de leite-PL e 0,25 para a produção de gordura-PG), seguido do nível alto (0,31 para $\mathrm{PG})$ e do nível baixo (0,38 para PL e 0,35 para PG). Rorato et al. (2000), em estudo semelhante, com a mesma raça no Brasil, encontraram maiores herdabilidades para PL no nível baixo $(0,58)$, seguida pelos níveis alto $(0,32)$ e médio $(0,26)$.

R. Bras. Zootec., v.34, n.2, p.514-519, 2005 
Marion et al. (2001), por sua vez, em animais da raça Holandesa, no RS, em diferentes níveis de produção e classes de desvios-padrão, registraram estimativas de herdabilidade nos níveis médio (0,20 para PL e $0,18$ para $P G)$, baixo $(0,19$ para PL e 0,17 para $P G)$ e alto $(0,18$ para PL e 0,15 para $P G)$. Torres et al. (2000), em animais da mesma raça, no Brasil, em níveis baixo, médio e alto para as classes de desviospadrão, relataram estimativas de herdabilidade para a PL de 0,29 no nível médio, 0,27 no nível baixo e 0,25 no nível alto, sendo as correlações de 0,97 entre os níveis baixo e médio, 0,89 entre o baixo e o alto e 0,91 entre os níveis médio e alto.

Este trabalho foi conduzido para estudar o comportamento das variâncias e estimar coeficientes de herdabilidade para os diferentes níveis de produção de leite e de gordura e as correlações genéticas entre estas características, para a raça Holandesa, no estado do Rio Grande do Sul.

\section{Material e Métodos}

Os registros de produção de leite e de gordura utilizados para o desenvolvimento deste estudo foram fornecidos pelo Serviço de Controle Leiteiro da Associação dos Criadores de Gado Holandês do Rio Grande do Sul. Os arquivos analisados continham informações de 8.887 registros de produção, de primeira a quinta ordem de lactação, de 5.875 vacas da raça Holandesa, filhas de 1.125 touros, no período desde 1984 a 2001.

Foram impostas algumas restrições nos arquivos originais visando aumentar a consistência dos dados, utilizando-se somente produções de animais com idades ao primeiro parto inferiores a 36 meses. Foram também descartadas as informações de lactações anormais, com período inferior a 100 dias. Rebanhos com menos de 10 observações e touros com somente uma filha, no mesmo nível de produção, foram excluídos. Foram criadas duas épocas de parição: a primeira correspondente ao período de outubro até março, e a segunda, de abril até setembro.

A edição dos dados, as análises preliminares, o agrupamento dos rebanhos por nível de produção e a concatenação das variáveis rebanho dentro do núcleo e época dentro do ano foram efetuados utilizando-se o do pacote estatístico SAS, versão 6.06 (SAS, 1995) para microcomputadores.

Para o estudo da heterogeneidade das variâncias e as estimativas dos coeficientes de herdabilidade, os dados foram estratificados em diferentes níveis, de acordo com a média de produção do rebanho. Foram formados três níveis: baixo $(\leq 6000 \mathrm{~kg})$, médio (>6000 a $\leq 7500 \mathrm{~kg}$ ) e alto (>7500 kg), de modo que cada rebanho só permanecesse em um nível. A determinação dos valores dos limites para os diferentes níveis foi definida considerando os conceitos de alta, média e baixa produção, vigentes na região e, também com o objetivo de distribuir os dados de forma mais uniforme. Os componentes de (co)variância e a correlação genética entre as características produtivas foram obtidos pelo método da Máxima Verossimilhança Restrita (Boldman et. al.,1995), utilizando um modelo animal incluindo como aleatórios os efeitos genético direto do animal (g), de ambiente permanente (pe) e o residual e, como efeitos fixos, rebanho dentro do núcleo de controle leiteiro, ano/época do parto, ordem da lactação e, como covariáveis, idade da vaca ao parto e duração da lactação.

O modelo bi característica proposto para o estudo, em termos matriciais, é descrito a seguir:

$$
\mathrm{Y}=\mathrm{X} \beta+\mathrm{Zg}+\mathrm{Wpe}+\mathrm{e}
$$

em que: $\mathrm{Y}=$ vetor das variáveis dependentes (produção de leite e gordura); $X=$ matriz de incidência associando $\beta$ com $Y ; \beta=$ vetor dos efeitos fixos, incluindo ano dentro de época do parto; ordem de lactação, rebanho dentro de núcleo e como covariáveis a idade da vaca, em dias e a duração da lactação, em dias (lineares e quadráticas); $\mathrm{Z}=$ matriz de incidência associando g com $\mathrm{Y} ; \mathrm{g}=$ vetor dos efeitos aleatórios do valor genético aditivo direto do animal; $\mathrm{W}=$ matriz de incidência associando pe com Y; pe = vetor dos efeitos aleatórios de ambiente permanente; $\mathrm{e}=$ vetor dos efeitos residuais.

Os componentes de (co)variâncias e os parâmetros genéticos e não-genéticos foram estimados pelo método da máxima verossimilhança restrita (REML), utilizando-se o aplicativo MTDFREML (Boldman et al., 1995). Adotou-se como critério de convergência a variância do Simplex inferior a $1 \times 10^{-6}$.

\section{Resultados e Discussão}

As médias observadas e respectivos desviospadrão para produção de leite (PL) e produção de gordura (PG) aos 305 dias de lactação, no arquivo completo e nos diferentes níveis estudados, encontram-se na Tabela 1. 
A média observada neste trabalho $(6.698 \mathrm{~kg} /$ lactação), para PL, para o arquivo completo, é superior aos valores relatados por Marion et. al. (2001) e Rorato et al. (2002) e similar ao valor encontrado por Durães et al. (2001), para a raça Holandesa no Brasil, de 5.083 a 6.876 kg/lactação. A média obtida neste trabalho é também superior àquela relatada por Araújo et al. (2002), para a raça Pardo-Suíça, no Brasil (5.941 kg/lactação).

Para PG, a média observada neste estudo (235 kg/ lactação) é superior àquelas encontradas, no Brasil, por Durães et al. (2001), Marion et. al. (2001) e Rorato et al. (2002), para a raça Holandesa, e por Araújo et al. (2002), para animais da raça Pardo-Suíça, que relataram valores de 172,6 a $228 \mathrm{~kg}$. Os resultados sugerem que a superioridade produtiva da população estudada, em relação à bibliografia consultada, deve ser decorrente da adoção de tecnologias de manejo e utilização de material genético de melhor qualidade.

As estimativas dos componentes de variância genética e de ambiente, para o arquivo completo e para os três níveis de produção dos rebanhos, encotram-se na Tabela 2. Observam-se variâncias heterogêneas, que cresceram com o aumento do nível de produção, tendência que corrobora os relatos de Hill et al. (1983), Dong \& Mao (1990), Mirande \& Van Vleck (1985) e Valencia et al. (1998), que observaram relação positiva e crescente entre os níveis de produção de leite e os componentes de variância.

O valor dos componentes de variância para a produção de gordura seguiu a mesma tendência que aqueles para produção de leite.

Os componentes de variância são maiores nos

Tabela 1- Médias de produção de leite (PL) e de gordura (PG) com respectivos desvios-padrão, entre parênteses, no arquivo completo e nos diferentes níveis analisados

Table 1 - Means and standard deviations for milk (PL) and fat $(P G)$ yields in complete file at different levels

\begin{tabular}{lccc}
\hline & $\mathrm{N}$ & $\mathrm{PL}(\mathrm{kg})$ & $\mathrm{PG}(\mathrm{kg})$ \\
\hline $\begin{array}{l}\text { Arquivo completo } \\
\begin{array}{l}\text { Complete file } \\
\text { Nível baixo }\end{array}\end{array}$ & 8.887 & $6.698(1.927)$ & $235(71)$ \\
$\begin{array}{l}\text { Low level } \\
\text { Nível médio }\end{array}$ & 2.981 & $4.922(742,80)$ & $163(30)$ \\
$\begin{array}{l}\text { Medium level } \\
\text { Nível alto }\end{array}$ & 2.584 & $6.715(427,25)$ & $225(25)$ \\
High level & 3.322 & $8.760(125,58)$ & $298(53)$ \\
\hline
\end{tabular}

$\mathrm{N}=$ número de observação (number of observation). níveis mais altos de produção, provavelmente, em conseqüência das melhores condições de meio proporcionadas nestes níveis, que permitiram melhor expressão do potencial genético dos animais. Nos níveis mais baixos de produção, o meio ambiente menos favorável pode não ter permitido, de forma mais eficiente, a expressão do potencial genético dos animais, comprimindo, dessa forma, a variância genética. Os efeitos da heterogeneidade de variâncias sobre a resposta à seleção dependem das diferenças entre as variâncias genéticas aditivas nos diferentes níveis e de suas relações com as variâncias fenotípicas. Dessa forma, a seleção pelo desempenho, sem considerar a variabilidade dentro do grupo, seria falha, pois, se a seleção for intensa, os grupos com animais com maiores valores genéticos contribuiriam com a maior parte dos reprodutores. Entretanto, se a heterogeneidade das variâncias não foi considerada, os valores genéticos destes reprodutores podem ter sido superestimados, em consequiência da maior variância genética aditiva e, conseqüentemente, do coeficiente de herdabilidade de maior magnitude, o

Tabela 2 - Componentes de variância genética $\left(\sigma^{2}{ }_{g}\right)$, de ambiente $\left(\sigma_{e}^{2}\right)$ para as características de produção de leite (PL) e de gordura (PG), para o arquivo completo e em diferentes níveis de produção

Table 2 - Genetic $\left(\sigma^{2} g\right)$, and environment $\left(\sigma^{2}{ }_{e}\right)$ variance components, for milk $(P L)$ and fat $(P G)$ yields at complete file and at different levels

\begin{tabular}{lccc}
\hline $\begin{array}{l}\text { Componente } \\
\text { de variância } \\
\text { Variance } \\
\text { component }\end{array}$ & \multicolumn{3}{c}{$\begin{array}{c}\text { Característica } \\
\text { Characteristic }\end{array}$} \\
\cline { 2 - 3 } & $\mathrm{PL}$ & $\mathrm{PG}$ \\
\hline \multicolumn{3}{c}{$\begin{array}{c}\text { Arquivo completo } \\
\text { Completefile }\end{array}$} \\
$\sigma_{\mathrm{g}}^{2}$ & 8.898 & 503 \\
$\sigma_{\mathrm{e}}^{2}$ & 932 & Nível baixo & 436 \\
& \multicolumn{3}{c}{ Low level } \\
$\sigma_{\mathrm{g}}^{2}$ & 488 & 95 \\
$\sigma_{\mathrm{e}}^{2}$ & 2.736 & Nível médio \\
$\sigma_{\mathrm{g}}^{2}$ & 892 & Mediun level & 452 \\
$\sigma_{\mathrm{e}}^{2}$ & 5.582 & & 182 \\
$\sigma_{\mathrm{g}}^{2}$ & 2.583 & Nível alto & 917 \\
$\sigma_{\mathrm{e}}^{2}$ & 9.432 & High level & 418 \\
\hline
\end{tabular}

R. Bras. Zootec., v.34, n.2, p.514-519, 2005 
que poderia ilustrar o caso típico observado na bovinocultura de leite, com grande comercialização de animais selecionados para alta produção em ambientes diferentes.

As estimativas de herdabilidade para os diferentes níveis de produção dos rebanhos estão apresentadas na Tabela 3. Os maiores coeficientes de herdabilidade ocorreram no nível alto e os menores, no nível médio de produção, sendo levemente inferiores do que àqueles estimados para o nível baixo, estando de acordo com os relatados por Boldman \& Freeman (1990), que estimaram coeficientes de herdabilidade de maior magnitude para o nível alto de produção, contrariando, porém, os obtidos por Marion et al. (2001), que obtiveram estimativas de herdabilidade maiores para o nível de produção baixo, seguido pelo alto e o médio.

Os coeficientes de herdabilidade para a população completa foram 0,22, para PL e 0,36, para PG. Este parâmetro para PL foi o mesmo relatado por Balieiro et al. (2000), que trabalharam com animais da raça Gir Leiteiro, e se assemelha àquele relatado por Ferreira \& Fernandes (2000) e por Marion et al. (2001), ambos para a raça Holandesa.

O valor da estimativa do coeficiente de herdabilidade para PG é superior aos valores relatados por Almeida et al. (1995), Ferreira \& Fernandes (2000), Balieiro et al. (2000) e Marion et al. (2001).

As estimativas de herdabilidade para PL e PG heterogêneas, para os diferentes níveis de produção, sugerem que a resposta esperada pelo uso de reprodutores superiores pode ser diferente, dependendo do ambiente a que suas filhas foram submetidas.

Tabela 3 - Coeficientes de herdabilidade para as características de produção de leite $(P L)$ e de gordura $(P G)$ e correlações genéticas ( $P L x$ $P G)$ entre elas para os diferentes níveis de produção

Table 3 - Heritability coefficients for milk (PL) and fat (PG) yield and genetic correlation (PL $\times$ PG) between then for the different levels

\begin{tabular}{lccc}
\hline & PL & PG & PLx PG \\
\hline $\begin{array}{l}\text { Arquivo completo } \\
\begin{array}{l}\text { Complete file } \\
\text { Nível baixo }\end{array}\end{array}$ & 0,22 & 0,36 & 0,98 \\
$\begin{array}{l}\text { Low level } \\
\text { Nível médio }\end{array}$ & 0,14 & 0,17 & 0,91 \\
$\begin{array}{l}\text { Medium level } \\
\text { Nível alto }\end{array}$ & 0,13 & 0,16 & 0,92 \\
High level & 0,19 & 0,21 & 1,00 \\
\hline
\end{tabular}

R. Bras. Zootec., v.34, n.2, p.514-519, 2005
O valor do coeficiente de correlação genética estimado entre PL e PG, no arquivo completo, foi 0,98, semelhante ao obtido por Matos et al. (1996), para a raça Holandesa, no Rio Grande do Sul. Para os diferentes níveis de produção de rebanhos, as correlações genéticas entre PL e PG foram 0,91, 0,92 e 1,00, respectivamente, para os níveis baixo, médio e alto, sugerindo que os genes responsáveis pela expressão de PL são praticamente, os mesmos para PG e que o melhoramento obtido para uma delas será transferido, quase que em sua totalidade para a outra.

\section{Conclusões}

A heterogeneidade observada nos componentes de variância e, conseqüentemente, nos coeficientes de herdabilidade sugerem que o resultado esperado como conseqüência da seleção, poderá não se confirmar nos diferentes níveis, comprometendo, portanto, a eficiência da seleção e frustrando a expectativa de ganho genético do produtor. Portanto, no ato de escolha do sêmen e/ou reprodutores, este fato deve ser considerado.

Os coeficientes de herdabilidade, embora relativamente baixos, indicam ser possível algum ganho genético, por meio da seleção, para as características estudadas.

As altas correlações genéticas observadas sugerem que a seleção para a produção de leite promove aumento quase equivalente na produção de gordura.

\section{Literatura Citada}

ALMEIDA, R.; RIBAS, N.P.; MONARDES, H.G. Estudo de características produtivas em rebanhos Holandeses em primeira cria na região da Batavo, Paraná. In: REUNIÃO ANUAL DA SOCIEDADE BRASILEIRA DE ZOOTECNIA, 32., 1995, Brasília. Anais... Brasília: Sociedade Brasileira de Zootecnia, 1995. p.692-694.

ARAÚJO, C.V.; TORRES, R.A.; RENNÓ, F.P. et al. Heterogeneidade de variância na avaliação genética de reprodutores da raça Pardo-Suíça no Brasil. Revista Brasileira de Zootecnia, v.31, n.3, p.1343-1349, 2002.

BALIEIRO, E.S.; PEREIRA, J.C.C.; VALENTE, J.et al. Estimativas de parâmetros genéticos e de tendências fenotípica, genética e de ambiente de algumas características produtivas da raça Gir leiteiro. Arquivo Brasileiro de Medicina Veterinária e Zootecnia, v.52, n.3, p.266-275, 2000.

BASTOS, J.F.P.; LÔBO, R.B.; SCHWENBER, E.B. et al. Mudanças genéticas, fenotípicas e de ambiente nas características produtivas em um rebanho da raça Pitangueiras. In: REUNIÃO ANUAL DA SOCIEDADE BRASILEIRA DE ZOOTECNIA, 35., 1998, Botucatu. Anais... Botucatu: Sociedade Brasileira de Zootecnia, 1998. p.482-484. 
BOLDMAN, K.G.; FREEMAN, A.E. Ajustment for heterogeneity of variances by herd production level in dairy cow and sire evaluation. Journal of Dairy Science, v.73, p.503-512, 1990.

BOLDMAN, K.G.; KRIESE. L.A.; Van VLECK, L.D. et al. A manual for use of MTDFREML - A set of program to obtain estimates of variances and covariances (DRAFT). Lincoln: Departament of Agriculture/Agricultural Research Service, 1995. 120p.

DONG, M.C.; MAO, I.L. Heterogeneity of (co)variance and herdability an different levels of intraherd milk production variance and of herd average. Journal of Dairy Science, v.73, p.843-845, 1990.

DURÃES, M.C.; FREITAS, A.F.; VALENTE, J. et al. Tendência genética para produção de leite e de gordura em rebanhos leiteiros da raça Holandesa no estado de Minas Gerais. Revista Brasileira de Zootecnia, v.30, n.1, p.66-70, 2001.

FERREIRA, G.B.; FERNANDES, H.D. Parâmetros genéticos para características produtivas em bovinos da raça Holandesa no estado de Goiás. Revista Brasileira de Zootecnia, v.29, n.2, p.421-426, 2000.

HILL, W.G.; EDWARDS, R.M.; AHMED, M.K.A. et al. Heritability of milk yield and composition at different levels and variability of production. Animal Production, v.36, n.1, p.59-68, 1983.

LEE, K.J.; PARK, D.K. Hetegeneity of milk production levels in Korean dairy herds. In: WORLD CONGRESSON GENETICS APLLIED TOLIVESTOCK PRODUCTION, 5., 1994, Ontário. Proceedings... Ontário: 1994. v.17, p.46-49.

LOFGREN, D.L.; VINSON, W.E.; PEARSON, R.E. et al. 1985. Herdability of milk yield at different herd means and variance for production. Journal of Dairy Science, v.68, n.10, p.2737-2739, 1985.

MATOS, R.S.; RORATO, P.R.N.; FERREIRA, G.B.B. et al. Estimativa de parâmetros genéticos para as produções de leite e gordura de bovinos da raça Holandesa no Rio Grande do Sul. In: JORNADA INTEGRADA DE PESQUISA, extensão e ensino, 03, 1996, Santa Maria. Anais... Santa Maria: Universidade Federal de Santa Maria, 1996. p.679.

MARION, A.E.; RORATO, P.R.N.; FERREIRA, G.B. et al. Estudo da heterogeneidade das variâncias para as características produtivas de rebanhos da raça Holandesa no Rio Grande do Sul. Revista Brasileira de Zootecnia, v.30, n.6S, p.1995-2001, 2001.
MIRANDE, S.L.; Van VLECK, L.D. Trends in genetic and phenotypic variances for milk production. Journal Dairy Science, v.68, n.9, p.2278-2286, 1985.

RAMOS, A.A.; VALENCIA, E.F.T.; WECHSLER, F.S. et al. Heterogeneidade da variância das características produtivas de bovinos da raça Holandesa nos trópicos. I - Estratificação por nível de produção de rebanho. In: REUNIÃO ANUAL DA SOCIEDADE BRASILEIRA DE ZOOTECNIA, 33., 1996, Fortaleza. Anais... Fortaleza: Sociedade Brasileira de Zootecnia, 1996. p.71-73.

RORATO, P.R.N.; Van VLECK, D.; VERNEQUE, R.S. et al. Interação genótipo-ambiente para a produção de leite em rebanhos da raça Holandesa no Brasil. 2. Uso de um modelo animal. Revista Brasileira de Zootecnia, v.29, n.6, p.2030-2035, 2000 (Suplemento 1)

RORATO, P.R.N.; EVERLING, D.M.; VARGAS, A.D.F. et al. Estudo da tendência genética para as características de produção e de qualidade do leite em rebanhos da raça Holandesa no estado do Rio Grande do Sul. In: REUNIÃO ANUALDA SOCIEDADE BRASILEIRA DE ZOOTECNIA, 39., 2002, Recife. Anais... São Paulo: Sociedade Brasileira de Zootecnia/Gnosis, [2002]. CD-ROM. Melhoramento Animal.

STATISTICAL ANALYSES SYSTEM - SAS. SAS User's guide: basic \& statistics. Cary: 1995.

STANTON, T.L.; BLAKE, R.W.; QUASS, R.L. et al. Genotype by enviroment interacion for Holstein milk yield in Colômbia, México and Porto Rico. Journal of Dairy Science, v.74, n.5, p.1700-1714, 1991.

TORRES, R.A.; BERGMANN, J.A.G.; COSTA, C.N. et al. Heterogeneidade de variância e avaliação genética de bovinos da raça Holandesa no Brasil. Revista Brasileira de Zootecnia, v.29, n.4, p.1050-1059, 2000.

VALENCIA, E.T.; RAMOS, A.A.; WESCHLER, F.S. et al. Heterogeneidade dos componentes de variância, na produção de leite de rebanhos dos estados de São Paulo e Paraná. In: REUNIÃO ANUAL DA SOCIEDADE BRASILEIRA DE ZOOTECNIA, 35., 1998, Botucatu. Anais... Botucatu: Sociedade Brasileira de Zootecnia, 1998. p.488-490.

Recebido em: 28/10/03 Aceito em: 23/11/04 